pain in $7(18.4 \%)$. Gastrointestinal (Gl) symptoms comprised of regurgitation in $31(81.5 \%)$ and dysphagia in $14(36.8 \%)$. Interstitial lung disease was present in $30(78.9 \%)$ patients, with higher prevalence in diffuse scleroderma $(100 \%)$ than in limited scleroderma $(70 \%)(p=0.01)$. Pulmonary hypertension was present in 18 patients, with significantly higher prevalence in diffuse disease $(57.1 \%)$, that was secondary to interstitial lung disease and in limited disease it was found in $(11.8 \%)(p<0.01)$. Thirty $(78.9 \%)$ patients were found to have restricted disease on pulmonary function tests.

Obstetric history showed a higher prevalence of primary infertility in at least 6 $(15.8 \%)$ patients, with significantly higher prevalence in limited systemic sclerosis disease as compared to diffuse disease $(23.5 \%$ vs $9.5 \%$, and $p=0.05)$. Fibromyalgia diagnosed as per ACR criteria was present in 7 (18.4\%) patients, and depression assessed by Hospital Anxiety and depression (HADS) score was present in $10(26.3 \%)$ patients.

Anti nucleic acid antibody (ANA) was found positive in $30(78.9 \%)$ patients. Anti Scl-70 antibodies were in $24(63.2 \%)$ patients, with significant association with diffuse disease ( $85 \%$ vs $35.3 \%$ and $p<0.01$ ), while anti centromere antibodies were present in 20 (52.6\%) patients; significantly higher in limited disease $(94.2 \%$ vs $19.0 \%$, and $p<0.01)$.

Conclusion: Scleroderma is a very important, autoimmune multisystem disease. It has female preponderence. Raynaud phenomenon is the most initial clinical feature followed by other manifestations of variable course and disease severity. Interstitial lung disease and pulmonary hypertension were the most important complication found in our patients which has poor prognosis. So, It is imperative to early diagnose and treat the disease manifesations to prevent future complications.

Keywords: Scleroderma, Systemic sclerosis.

DISCLOSURE OF INTEREST:

Sadia Asif: None declared, Muhammad Haroon: None declared, Dr Asadullah Khan: None declared, Dr. Muhammad Faiq: None declareDOI: 10.1136/ annrheumdis-2021-eular.1635

\section{AB0094 INCREASE OF ENDOTHELIAL PROGENITOR CELLS IN SYSTEMIC SCLEROSIS-ASSOCIATED INTERSTITIAL LUNG DISEASE}

V. Pulito-Cueto $^{1}$, S. Remuzgo Martinez ${ }^{1}$, F. Genre ${ }^{1}$, B. Atienza-Mateo ${ }^{1,2,3}$, V. M. Mora-Cuesta ${ }^{1,4}$, D. Iturbe-Fernández ${ }^{1,4}$, L. Lera-Gómez ${ }^{1}$, R. PérezFernández ${ }^{1}$, D. Prieto-Peña ${ }^{1,3}$, V. Portilla ${ }^{1,3}$, R. Blanco ${ }^{1,3}$, A. Corrales ${ }^{1,3}$, J. M. Cifrián-Martínez ${ }^{1,4,5}$, R. López-Mejías ${ }^{1}$, M. A. González-Gay ${ }^{1,3,5,6}{ }^{1}{ }^{1}$ IDIVAL, Research Group on Genetic Epidemiology and Atherosclerosis in Systemic Diseases and in Metabolic Bone Diseases of the Musculoskeletal System, Santander, Spain; ${ }^{2}$ Hospital Universitario Marqués de Valdecilla, 'López Albo Post-Residency Programme, Santander, Spain; ${ }^{3}$ Hospital Universitario Marqués de Valdecilla, Department of Rheumatology, Santander, Spain; ${ }^{4}$ Hospital Universitario Marqués de Valdecilla, Department of Pneumology, Santander, Spain; ${ }^{5}$ Universidad de Cantabria, School of Medicine, Santander, Spain; ${ }^{6}$ Faculty of Health Sciences, University of the Witwatersrand, Cardiovascular Pathophysiology and Genomics Research Unit, School of Physiology, Johannesburg, South Africa

Background: Endothelial progenitor cells (EPC), involved in vasculogenesis and endothelial tissue repair, have been described as relevant players in vascular and connective tissue diseases [1-2]. In this regard, a previous study of our group disclosed that the degree of EPC frequency may help to identify the presence of interstitial lung disease (ILD) in rheumatoid arthritis patients [3]. Given that ILD is the main cause of mortality in patients with systemic sclerosis (SSc) [1, 4-6], the understanding of the role of EPC in the mechanism of SSc-ILD+ vasculopathy is crucial.

Objectives: To assess the potential role of EPC on vascular dysfunction associated with the presence of ILD in patients with SSc.

Methods: Peripheral venous blood was collected from a total of 39 patients with SSc, 20 with ILD (SSc-ILD ${ }^{+}$) and 19 without ILD (SSc-ILD'). All subjects were recruited from the Rheumatology and Pneumology departments of Hospital Universitario Marqués de Valdecilla, Santander, Spain. Quantification of EPC was analyzed by flow cytometry. EPC were considered as CD $34^{+}, \mathrm{CD}^{\mathrm{LOw}}{ }^{\mathrm{Low}}, \mathrm{CD} 09^{+}$ and $\mathrm{CD} 133^{+}$

Results: Statistically significant differences in EPC frequency between patients with SSc-ILD ${ }^{+}$and patients with SSc-ILD were disclosed. Specifically, an increase of EPC frequency was observed in SSc-ILD ${ }^{+}$patients when compared to patients with SSc-ILD' (mean \pm standard deviation: $0.033 \pm 0.012$ versus 0.021 \pm 0.017 , respectively, $p=0.012$ ).

Conclusion: Our results suggest a potential role of EPC on vascular damage associated with the manifestation of ILD in patients with SSc.

REFERENCES:

[1] Eur J Rheumatol 2020;7(Suppl 3):S139-S146.

[2] Arthritis Rheum 2009;60(11):3168-79.

[3] J Clin Med 2020;9(12):4098.
[4] Ann Rheum Dis 2007;66(7):940-4.

[5] Rheumatology (Oxford) 2010;49(12):2375-80.

[6] Eur Respir Rev 2015;24(135):102-14.

Acknowledgements: Personal funds, VP-C: PREVAL18/01 (IDIVAL); SR-M: RD16/0012/0009 (ISCIII-ERDF); LL-G: INNVAL20/06 (IDIVAL); RP-F: START PROJECT (FOREUM); RL-M: Miguel Servet type I CP16/00033 (ISCIII-ESF).

Disclosure of Interests: Verónica Pulito-Cueto: None declared, Sara Remuzgo Martinez: None declared, Fernanda Genre: None declared, Belén Atienza-Mateo: None declared, Victor Manuel Mora-Cuesta: None declared, David Iturbe-Fernández: None declared, Leticia Lera-Gómez: None declared, Raque Pérez-Fernández: None declared, Diana Prieto-Peña: None declared, Virginia Portilla: None declared, Ricardo Blanco Speakers bureau: Abbvie, Pfizer, Roche Bristol-Myers, Janssen and MSD, Consultant of: Abbvie, Pfizer, Roche, Bristol-Myers, Janssen and MSD, Grant/research support from: Abbvie, MSD and Roche, Alfonso Corrales: None declared, Jose Manuel Cifrián-Martínez: None declared, Raquel López-Mejías: None declared, Miguel A González-Gay Speakers bureau: Pfizer, Abbvie, MSD, Grant/research support from: Pfizer, Abbvie, MSD

DOI: 10.1136/annrheumdis-2021-eular.2350

\section{$\mathrm{AB} 0095$}

EXPRESSION AND PATHOGENIC ROLES OF INTEGRIN FAMILY GENE IN SYSTEMIC SCLEROSIS

D. $\mathrm{Xu}^{1}, \mathrm{~T} . \mathrm{Li}^{1}, \mathrm{R} . \mathrm{Mu}^{1} .{ }^{1}$ Peking University Third Hopsital, Department of Rheumatology and Immunology, Beijing, China

Background: Emerging evidence have shown that some integrin members are associated with inflammation and fibrosis in systemic sclerosis (SSc) patients ${ }^{[1-2]}$ However, the expression patterns and pathogenic significance of the whole integrin family in SSc are still unclear.

Objectives: This study aimed at evaluating the integrin family gene expression in skin lesion from SSc patients and exploring its potential pathogenic mechanism. Methods: We utilized the public datasets of SSc skin tissue from Gene Expression Omnibus (GEO) database to analyze the expression and clinical significance of integrin family genes in SSc. In addition, functional enrichment and pathway analysis were also conducted.

Results: Compared with healthy controls, ITGA5, ITGA7, ITGA8, ITGB2, ITGB5, ITGAE and ITGB3BP were abnormally overexpressed in the skin of SSc. Further analysis indicated that ITGA5, ITGA7, ITGA8, ITGB2 and ITGB5 were positively correlated with modified Rodnan skin thickness score (mRSS), while ITGAE and ITGB3BP were negatively correlated with $\mathrm{mRSS}$ in SSc. Increased ITGB5 expression was associated with positive of anti-centromere antibody (ACA) Functional enrichment and pathway analysis showed that integrin members had multiple functions in SSc. Among them, ITGA5, ITGB2 and ITGB5 might synergistically promote SSc through affecting extracellular matrix (ECM) turn over, ECM-receptor interaction, focal adhesion and leukocyte trans-endothelial migration. ITGA5 and ITGB5 also affected angiogenesis and endothelial cell function. In addition, ITGA5 was uniquely enriched for actin organization, ITGB5 was uniquely enriched for TGF- $\beta$ signaling, and ITGB2 was uniquely associated with immune cells activation.

Conclusion: Our results implied that integrins, especially ITGA5, ITGB5, ITGB2 participated in the process of inflammation, vasculopathy and fibrosis in SSc. Together, they might render important therapeutic targets for SSc.

\section{REFERENCES:}

[1] Brown M, O'Reilly S. The immunopathogenesis of fibrosis in systemic sclerosis. Clin Exp Immunol. 2019;195(3):310-321.

[2] Gerber, E.E., et al., Integrin-modulating therapy prevents fibrosis and autoimmunity in mouse models of scleroderma. Nature, 2013. 503(7474): $p$ 126-30.

Disclosure of Interests: None declared

DOI: 10.1136/annrheumdis-2021-eular.3646

\section{Vasculitis - aetiology, pathogenesis and animal models}

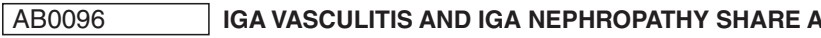 SIMILAR IL17A ASSOCIATION PATTERN}

D. Prieto-Peña ${ }^{1}$, F. Genre ${ }^{1}$, S. Remuzgo Martinez ${ }^{1}$, V. Pulito-Cueto ${ }^{1}$, B. AtienzaMateo $^{1,2}$, B. Sevilla ${ }^{3}$, J. Llorca ${ }^{4}$, N. Ortego ${ }^{5}$, L. Lera-Gómez ${ }^{1}$, M. Leonardo ${ }^{6}$,

A. Peñalba ${ }^{6}$, L. Martín-Penagos ${ }^{7}$, J. A. Miranda-Filloy ${ }^{8}$, J. Narváez ${ }^{9}$, L. Caminal Montero $^{10}$, P. Collado ${ }^{11}$, A. Fernandez-Nebro ${ }^{12}$, G. Díaz-Cordoves ${ }^{12}$,

S. Cigarrán ${ }^{13}$, J. Calviño ${ }^{14}$, C. Cobelo ${ }^{14}$, J. Sanchez Perez $^{15}$, D. De Argila ${ }^{15}$,

E. Rubio-Romero ${ }^{16}$, M. Leon Luque ${ }^{16}$, J. M. Blanco-Madrigal ${ }^{17}$, E. Galindez ${ }^{17}$,

J. Martin Ibanez ${ }^{18}$, S. Castañeda ${ }^{19}$, R. Blanco ${ }^{1}$, M. A. González-Gay ${ }^{1,20,21}$

R. López-Mejías ${ }^{1} .{ }^{1}$ Research Group on Genetic Epidemiology and 
Atherosclerosis in Systemic Diseases and in Metabolic Bone Diseases of the Musculoskeletal System, Division of Rheumatology, Hospital Universitario Marqués de Valdecilla, IDIVAL, Santander, Spain; ${ }^{2}$ López Albo' Post-Residency Programme, Hospital Universitario Marqués de Valdecilla, Santander, Spain; ${ }^{3}$ Hospital Universitario San Cecilio, Division of Pediatrics, Granada, Spain; ${ }^{4}$ Department of Epidemiology and Computational Biology, School of Medicine, University of Cantabria, and CIBER Epidemiología y Salud Pública (CIBERESP), IDIVAL, Santander, Spain; ${ }^{5}$ Hospital Universitario San Cecilio, Systemic Autoimmune Diseases Unit, Granada, Spain; ${ }^{6}$ Hospital Universitario Marqués de Valdecilla, Division of Pediatrics, Santander, Spain; ${ }^{7}$ Hospital Universitario Marqués de Valdecilla, Division of Nephrology, Santander, Spain; ${ }^{8}$ Hospital Universitario Lucus Augusti, Division of Rheumatology, Lugo, Spain; ${ }^{9}$ Hospital Universitario de Bellvitge, Division of Rheumatology, Barcelona, Spain; ${ }^{10}$ Hospital Universitario Central de Asturias, Division of Rheumatology, Oviedo, Spain; ${ }^{11}$ Hospital Universitario Severo Ochoa, Division of Rheumatology, Madrid, Spain; ${ }^{12}$ Hospital Regional Universitario Carlos Haya, Division of Rheumatology, Málaga, Spain; ${ }^{13}$ Hospital da Costa Burela, Division of Nephrology, Lugo, Spain; ${ }^{14}$ Hospital Universitario Lucus Augusti, Division of Nephrology, Lugo, Spain; ${ }^{15}$ Hospital Universitario de La Princesa, Dermatology Department, Madrid, Spain; ${ }^{16}$ Hospital Universitario Virgen del Rocío, Division of Rheumatology, Sevilla, Spain; ${ }^{17}$ Hospital Universitario de Basurto, Division of Rheumatology, Bilbao, Spain; ${ }^{18}$ Instituto de Parasitolog ía y Biomedicina 'LópezNeyra', CSIC, PTS Granada, Granada, Spain; ${ }^{19}$ Hospital Universitario de La Princesa, IIS-Princesa, Division of Rheumatology, Madrid, Spain; ${ }^{20}$ University of Cantabria, School of Medicine, Santander, Spain; ${ }^{21}$ University of the Witwatersrand, Cardiovascular Pathophysiology and Genomics Research Unit, School of Physiology, Faculty of Health Sciences, Johannesburg, South Africa

Background: $\lg \mathrm{A}$ vasculitis ( $\lg \mathrm{AV})$ and $\lg \mathrm{A}$ nephropathy $(\operatorname{Ig} \mathrm{AN})$ are inflammatory conditions that share pathogenic and molecular mechanisms [1] and may represent different outcomes of a continuous spectrum of disease [2]. Interleukin (IL)17A has been identified as a common genetic risk locus for several immune-mediated diseases [3, 4].

Objectives: To determine whether IgAV and IgAN exhibit a different IL17A association pattern.

Methods: Five IL17A tag polymorphisms (rs4711998, rs8193036, rs3819024, rs2275913 and rs7747909) were genotyped in 388 Caucasian patients with IgAV, 99 patients with IgAN and 1,003 sex and ethnically matched healthy controls.

Results: No statistically significant differences between patients with IgAV and healthy controls and between patients with IgAN and healthy controls were observed when each IL17A genetic variant was analyzed independently (Table 1). Similarly, IgAV patients exhibited similar genotype and allele IL17A frequencies than those with IgAN (Table 1). Moreover, no genotype or allele differences between IgAV patients who developed nephritis and patients with IgAN were detected. Furthermore, haplotype frequencies were similar in patients with $\lg A V$, IgAV and nephritis and those with $\lg A N$.

Table 1. Genotype and allele frequencies of IL17A gene in patients with IgA vasculitis, patients with IgA nephropathy and healthy controls.

\begin{tabular}{|c|c|c|c|c|c|c|c|}
\hline Polymorphism & Change & Data set & $1 / 1$ & $1 / 2$ & $2 / 2$ & 1 & 2 \\
\hline \multirow[t]{3}{*}{ rs4711998 } & $\mathrm{G} / \mathrm{A}$ & IgAV & $53.4(207)$ & 38.9 (151) & $7.7(30)$ & $72.8(565)$ & $27.2(211)$ \\
\hline & & IgAN & $49.0(48)$ & $42.9(42)$ & $8.2(8)$ & 70.4 (138) & $29.6(58)$ \\
\hline & & Controls & 52.7 (529) & $41.2(413)$ & $6.1(61)$ & 73.3 (1471) & 26.7 (535) \\
\hline \multirow[t]{3}{*}{ rs8193036 } & T/C & $\lg A V$ & 57.0 (221) & 38.4 (149) & 4.6 (18) & 76.2 (591) & $23.8(185)$ \\
\hline & & IgAN & $64.3(63)$ & 31.6 (31) & $4.1(4)$ & 80.1 (157) & 19.9 (39) \\
\hline & & Controls & 60.3 (605) & 35.2 (353) & $4.5(45)$ & 77.9 (1563) & 22.1 (443) \\
\hline \multirow[t]{3}{*}{ rs3819024 } & $\mathrm{A} / \mathrm{G}$ & IgAV & $44.1(171)$ & 43.3 (168) & $12.6(49)$ & $65.7(510)$ & $34.3(266)$ \\
\hline & & IgAN & 39.4 (39) & $54.5(54)$ & $6.1(6)$ & 66.7 (132) & $33.3(66)$ \\
\hline & & Controls & 45.6 (457) & $44.6(447)$ & $9.9(99)$ & 67.8 (1361) & $32.2(645)$ \\
\hline \multirow[t]{3}{*}{ rs2275913 } & $\mathrm{G} / \mathrm{A}$ & IgAV & 44.6 (172) & $43.3(167)$ & $12.2(47)$ & $66.2(511)$ & $33.8(261)$ \\
\hline & & IgAN & $39.8(39)$ & $53.1(52)$ & $7.1(7)$ & $66.3(130)$ & $33.7(66)$ \\
\hline & & Controls & 44.8 (449) & $44.2(443)$ & 11.1 (111) & 66.8 (1341) & $33.2(665)$ \\
\hline \multirow[t]{3}{*}{ rs7747909 } & $\mathrm{G} / \mathrm{A}$ & IgAV & 53.9 (209) & 39.4 (153) & $6.7(26)$ & $73.6(571)$ & 26.4 (205) \\
\hline & & IgAN & 41.1 (39) & $54.7(52)$ & $4.2(4)$ & 68.4 (130) & $31.6(60)$ \\
\hline & & Controls & $53.0(532)$ & 39.4 (395) & $7.6(76)$ & 72.7 (1459) & $27.3(547)$ \\
\hline
\end{tabular}

Conclusion: Our results revealed that IgAV and IgAN share a similar IL17A association pattern.

REFERENCES:

[1] N Engl J Med 2013;368:2402-14.

[2] Am J Kidney Dis 1988;12:373-7.

[3] Ann Rheum Dis 2014;73:1742-5.

[4] Mediators Inflamm 2018;2018:1395823.

Acknowledgements: This study was supported by European Union FEDER funds and "Fondo de Investigaciones Sanitarias" (grant PI18/00042) from 'Instituto de Salud Carlos III' (ISCIII, Health Ministry, Spain). DP-P is a recipient of a Río Hortega programme fellowship from the ISCIII, co-funded by the European Social Fund (ESF, 'Investing in your future') [grant number CM20/00006];
SR-M is supported by funds of the RETICS Program co-funded by the European Regional Development Fund (ERDF) [grant number RD16/0012/0009]; VP-C is supported by a pre-doctoral grant from IDIVAL [grant number PREVAL 18/01]; BA-M is a recipient of a 'López Albo' Post-Residency Programme funded by Servicio Cántabro de Salud; LL-G is supported by funds of IDIVAL [grant numbe INNVAL20/06]; RL-M is a recipient of a Miguel Servet type I programme fellowship from the ISCIII, co-funded by the European Social Fund (ESF, 'Investing in your future') [grant number CP16/00033].

Disclosure of Interests: Diana Prieto-Peña: None declared, Fernanda Genre: None declared, Sara Remuzgo Martinez: None declared, Verónica Pulito-Cueto: None declared, Belén Atienza-Mateo: None declared, Belén Sevilla: None declared, Javier Llorca: None declared, Norberto Ortego: None declared, Leticia Lera-Gómez: None declared, Maite Leonardo: None declared, Ana Peñalba: None declared, Luis Martín-Penagos: None declared, Jose Alberto Miranda-Filloy: None declared, J. Narváez: None declared, LUIS CAMINAL MONTERO: None declared, PAZ COLLADO: None declared, Antonio Fernandez-Nebro: None declared, Gisela Díaz-Cordoves: None declared, Secundino Cigarrán: None declared, Jesús Calviño: None declared, Carmen Cobelo: None declared, Javier Sanchez Perez: None declared, Diego de Argila: None declared, Esteban Rubio-Romero: None declared, MANUEL LEON LUQUE: None declared, Juan María Blanco-Madrigal: None declared, E. Galindez: None declared, Javier Martin Ibanez: None declared, Santos Castañeda: None declared, Ricardo Blanco Speakers bureau: Abbvie, Pfizer, Roche, Bristol-Myers, Janssen and MSD, Consultant of: Abbvie, Pfizer, Roche, Bristol-Myers, Janssen and MSD, Grant research support from: Abbvie, MSD and Roche, Miguel A González-Gay Speak ers bureau: Pfizer, Abbvie, MSD, Grant/research support from: Pfizer, Abbvie, MSD, Raquel López-Mejías: None declared DOI: 10.1136/annrheumdis-2021-eular.766

\section{AB0097 \\ DIAGNOSTIC ACCURACY OF SERUM MARKERS IN LARGE VESSEL VASCULITIS AND CORRELATION WITH PET IMAGING}

R. Bilici Salman ${ }^{1}$, H. Satış2, A. Avanoglu Guler3, H. Karadeniz3, H. Küçük', S. Haznedaroglu' ${ }^{1}$ A. Tufan ${ }^{1}$, B. Goker ${ }^{1}$, U. O. Akdemir ${ }^{4}$, L. O. Atay ${ }^{4}$, H. Paşaoğlu ${ }^{5}$, M. A. Ozturk' ${ }^{1}{ }^{1}$ Gazi University School of Medicine, Rheumatology, Ankara, Turkey; ${ }^{1}$ Gazi University School of Medicine, Rheumatology, Ankara, Turkey; ${ }^{1}$ Gazi University School of Medicine, Rheumatology, Ankara, Turkey; ${ }^{4}$ Gazi University School of Medicine, Nuclear Medicine, Ankara, Turkey; ${ }^{5}$ Gazi University School of Medicine, Biochemistry, Ankara, Turkey

Background: The onset of symptoms in large-vessel vasculitis (LVV) tends to be subacute, which often leads to a delay in diagnosis, during which time vascular disease may start and progress to become symptomatic. PET/CT has been recognised since the 2003s as a promising tool in evaluating of LVV. There is no gold standard diagnostic laboratory tests for this patient group, novel markers for active LVV is needed.

Objectives: to investigate the association between vascular inflammation, as detected by PET imaging and interleukin-6 (IL-6), pentraxin3 (PTX3), and B-cell-activating-factor (BAFF) in subjects with LVV.

Methods: The study included 67 patients patients with newly diagnosed GCA $(n=27)$ or TA $(n=9)$ and healthy control $(n=31)$ who had been referred to the Rheumatology Unit at Gazi University, between December 2017 and August 2020. PET images obtained from an 29 patients (22 with GCA and 7 with TA) who had not received any corticosteroid treatment prior to PET imaging and blood sampling. IL-6, PTX3, and BAFF levels were determined quantitatively by enzyme-linked immunosorbent assay (ELISA) kits.

Results: 36 patients with LVV (20 females, 16 males; age $64,5 \pm 16,6)$ and $31 \mathrm{HC}$ (14 females, 17 males; age37,1 $\pm 9,6$ ) were analysed. Serum levels of IL-6,PTX3, BAFF, ESR and CRP are increased in patients with newly diagnosed LVV compared with those in control subjects. In a ROC analysis, serum IL-6 provided excellent discrimination of newly diagnosed LVV patients from $\mathrm{HC}$, as indicated by AUCs $>0.90$. Serum BAFF also accurately distinguished newly diagnosed LVV patients from $\mathrm{HC}$ with AUCs $>0.80$. Serum PTX3 did not provide an AUC $>0.80$. In this study, we correlate vascular inflammation, as detected by PET imaging in newly diagnosed LVV patients, with the ESR, CRP. PTX3, IL-6 and BAFF. As a result, none of these markers has been associated with vascular inflammation as measured using PET.

Conclusion: In conclusion, our study shows that serum levels of PTX3, IL- 6 and BAFF are increased in most LVV patients. The diagnostic value of BAFF and IL-6, both separately and in combination, should be further evaluated in larger cohorts of LVV patients, as well as in patients with infections or other inflammatory conditions. However, none of these markers has been associated with vascular inflammation as measured using PET.

Disclosure of Interests: None declared

DOI: 10.1136/annrheumdis-2021-eular.3719 